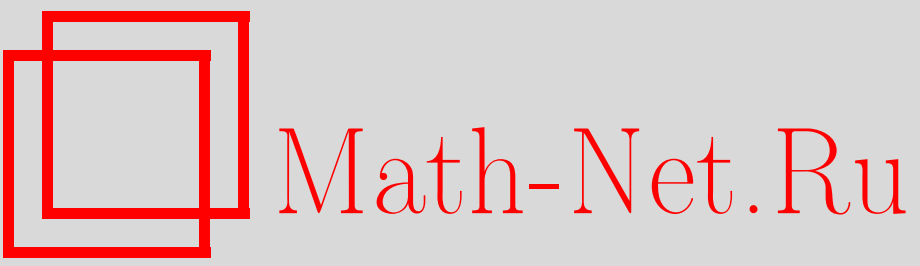

М. Х. Шерматов, О точечном взаимодействии двух различимых частиц во внешнем поле, ТМФ, 2000, том 125, номер 1, 74-90

DOI: https://doi.org/10.4213/tmf658

Использование Общероссийского математического портала Math-Net.Ru подразумевает, что вы прочитали и согласны с пользовательским соглашением

http://www . mathnet.ru/rus/agreement

Параметры загрузки:

IP : 54.80 .97 .219

26 апреля 2023 г., 16:41:55 
ТЕОРЕТИЧЕСКАЯ

И МАТЕМАТИЧЕСКАЯ

ФИЗИКА

Том 125, № 1

октябрь, 2000

(C) 2000 г.

\section{О ТОЧЕЧНОМ ВЗАИМОДЕЙСТВИИ ДВУХ РАЗЛИЧИМЫХ ЧАСТИЦ ВО ВНЕШНЕМ ПОЛЕ}

Рассматривается модель точечного взаимодействия двух частиц по внешнем поле, аналогичная модели Скорнякова-Тер-Мартиросяна и трактуемая с помощью теории самосопряженных расширений симметрических операторов. Показано, что соответствующий оператор энергии имеет бесконечный набор связанных состояний со значениями энергии, уходящими на $-\infty$.

\section{1. ВВЕДЕНИЕ}

В работе [1] было впервые предложено строгое математическое описание точечного взаимодействия в квантовой механике. Предложенный подход был проиллюстрирован на примере взаимодействия двух нерелятивистских скалярных частищ, и гамильтониан системы трактовался с помошью теории самосопряженных расширений симметрических операторов (см., например, [2]). В работах [3, 4] этот подход был применен к системе трех одинаковых квантовых частиц (бозонов), взаимодействующих точечным образом, и гамильтониан системы вводился как некоторое самосопряженное расширение симметрического оператора кинетической энергии трех частиц

$$
H_{0}=-\Delta_{x_{1}}-\Delta_{x_{2}}-\Delta_{x_{3}}
$$

определенного на области $D\left(H_{0}\right) \subset L_{2}^{s}\left(\left(\mathbb{R}^{3}\right)^{3}\right)$ функций трех переменных $x_{j} \in \mathbb{R}^{3}$, $j=1,2,3$, обрашающихся в нуль при совпадении любых двух аргументов $x_{j}=x_{k}$, $j \neq k, j, k=1,2,3$. Эта задача впервые рассматривалась в работе Скорнякова и ТерМартиросяна [5], где было указано одно из расширений оператора $H_{0}$ (обозначаемое через $H_{\varepsilon}, \varepsilon \in \mathbb{R}^{1}$ ), которое, как позже было показано в работах Данилова [6] и Минлоса, Фаддеева $[3,4]$, является лишь симметрическим, но не самосопряженным расширением $H_{0}$. В работе [3] показано, что оператор $H_{\varepsilon}$ имеет индексы дефекта $(1,1)$, и построены все самосопряженные расширения $H_{\varepsilon, \beta}$ оператора $H_{\varepsilon}$. При этом оказалось, что все эти расширения имеют неограниченный снизу дискретньй спектр (возможно “падение на центр"). В работе [7] с использованием результатов работы [3] изучался гамильтониан

\footnotetext{
* Самаркандское отделение АН Республики Узбекистан, Самарканд, Узбекистан
} 
системы трех частиц (двух фермионов и одной частицы иной природы), взаимодействуюших точечным образом. Этот гамильтониан вводился как расширение Скорнякова и Тер-Мартиросяна оператора $H_{0}$ в пространстве $L_{2}^{a(1,2)}\left(\left(\mathbb{R}^{3}\right)^{3}\right)$ функций, антисимметричных относительно переменных $x_{1}$ и $x_{2}$, и было показано, что эти расширения являются самосопряженными и полуограниченными. В работе [8] обобшены результаты работ $[3,4,7]$ на случай трех различимых частиц (обладающих даже различными массами)

В настоящей работе, следуя в основном схеме работ $[7,8]$, мы рассматриваем случай двух различимых частиц, взаимодействуюших точечным образом во внешнем поле (также с точечным потенциалом). В работе доказано сушествование бесконечного числа связанных состояний системы со значениями энергии, уходяшими на $-\infty$ (эффект Томаса).

\section{2. ПОСТАНОВКА ЗАДАЧИ}

Гамильтониан системы двух различимых квантовых частиц, взаимодействующих точечным образом во внешнем поле, задается как некоторое самосопряженное расширение симметрического оператора

$$
\widehat{H}=-\frac{1}{2 m_{1}} \Delta_{x_{1}}-\frac{1}{2 m_{2}} \Delta_{x_{2}}
$$

действуюшего в пространстве $L_{2} \equiv L_{2}\left(\left(\mathbb{R}^{3}\right)^{2}\right)$ волновых функций $\psi\left(x_{1}, x_{2}\right)$ и определенного на области

$$
D(\widehat{H})=\left\{\psi \in L_{2}: \Delta_{x_{j}} \psi \in L_{2}, \quad j=1,2 ; \quad \psi(x, x)=\psi(x, 0)=\psi(0, x)=0\right\} .
$$

Далее удобно перейти к импульсному представлению. В результате преобразования Фурье замыкание оператора $\widehat{H}$ перейдет в следуюший симметрический оператор:

$$
(H f)\left(p_{1}, p_{2}\right)=\left(\frac{1}{2 m_{1}} p_{1}^{2}+\frac{1}{2 m_{2}} p_{2}^{2}\right) f\left(p_{1}, p_{2}\right),
$$

область определения $D(H) \subset L_{2}$ которого состоит из функций $f\left(p_{1}, p_{2}\right)$, удовлетворяющих условиям

$$
H f \in L_{2}, \quad \int_{\left(\mathbb{R}^{3}\right)^{2}} f\left(p_{1}, p_{2}\right)\left[u_{1}\left(p_{1}\right)+u_{2}\left(p_{2}\right)+u_{3}\left(p_{1}+p_{2}\right)\right] d p_{1} d p_{2}=0,
$$

где $u_{j}(p)$ - произвольная функция, удовлетворяющая условию

$$
\int_{\mathbb{R}^{3}}\left|u_{j}(p)\right|^{2}\left(p^{2}+1\right)^{-\frac{1}{2}} d p<\infty, \quad j=1,2,3
$$

Поскольку оператор $H$ неотрицателен, естественно воспользоваться теорией расширений полуограниченных симметрических операторов. Используемые нами приемы можно найти в работе Бирмана [9]. С помошью условия (2) нетрудно убедиться, что 
дефектное подпространство $\Re_{-1}(H)=[\operatorname{Ran}(H+E)]^{\perp}$ оператора $H+E$ состоит из элементов вида

$$
\Phi_{\vec{u}}\left(p_{1}, p_{2}\right)=\left(u_{1}\left(p_{1}\right)+u_{2}\left(p_{2}\right)+u_{3}\left(p_{1}+p_{2}\right)\right)\left(\frac{1}{2 m_{1}} p_{1}^{2}+\frac{1}{2 m_{2}} p_{2}^{2}+1\right)^{-1},
$$

где $u_{1}, u_{2}, u_{3}$ - функции, удовлетворяющие условию (3). Заметим, что функция $\Phi_{\vec{u}}\left(p_{1}, p_{2}\right)$ вида (4) принадлежит пространству $\Re_{-1}(H)$ тогда и только тогда, когда функции $u_{1}, u_{2}, u_{3}$ удовлетворяют условию (3). Легко видеть, что пространство $\Re_{-1}(H)$ изоморфно гильбертову пространству $W^{(3)}$ троек функций $\vec{u}(p)=\left(u_{1}(p), u_{2}(p), u_{3}(p)\right)$, полученному пополнением пространства $L_{2}^{(3)} \equiv L_{2}\left(\mathbb{R}^{3}\right) \oplus L_{2}\left(\mathbb{R}^{3}\right) \oplus L_{2}\left(\mathbb{R}^{3}\right)$ по скалярному произведению $[\vec{u}, \vec{v}]=(K \vec{u}, \vec{v})$, где $(\cdot, \cdot)$ - скалярное произведение в $L_{2}^{(3)}, K$ - положительный ограниченньй оператор в $L_{2}^{(3)}$, действуюший по формулам

$$
\begin{aligned}
(K \vec{u})_{k}(p)= & \frac{\pi^{2}\left(2 m_{j}\right)^{\frac{3}{2}}}{\sqrt{\left(2 m_{l}\right)^{-1} p^{2}+1}} u_{k}(p)+\int_{\mathbb{R}^{3}} \frac{u_{j}(q)+u_{3}(p+q)}{\left(\left(2 m_{k}\right)^{-1} p^{2}+\left(2 m_{j}\right)^{-1} q^{2}+1\right)^{2}} d q, \\
& j, k=1,2, \quad j \neq k, \\
(K \vec{u})_{3}(p)= & \frac{\pi^{2} \eta^{\frac{3}{2}}}{\sqrt{(2 M)^{-1} p^{2}+1}} u_{3}(p)+\int_{\Gamma_{p}} \frac{u_{1}\left(p_{1}\right)+u_{2}\left(p_{2}\right)}{\left(\left(2 m_{1}\right)^{-1} p_{1}^{2}+\left(2 m_{2}\right)^{-1} p_{2}^{2}+1\right)^{2}} d v_{p} .
\end{aligned}
$$

Здесь $\Gamma_{p}=\left\{\left(p_{1}, p_{2}\right) \in\left(\mathbb{R}^{3}\right)^{2}: p_{1}+p_{2}=p\right\}, p \in \mathbb{R}^{3},-$ семейство многообразий с естественной лебеговской мерой $d v_{p}, M=m_{1}+m_{2}, \eta=2 m_{1} m_{2} /\left(m_{1}+m_{2}\right)$.

Лемма 1. Область определения $D\left(H^{*}\right)$ сопряжснного оператора $H^{*}$ состоит из функиий вида

$$
g\left(p_{1}, p_{2}\right)=f\left(p_{1}, p_{2}\right)+\Phi_{\vec{u}}\left(p_{1}, p_{2}\right)+\left(\frac{1}{2 m_{1}} p_{1}^{2}+\frac{1}{2 m_{2}} p_{2}^{2}+1\right)^{-1} \Phi_{\vec{v}}\left(p_{1}, p_{2}\right),
$$

əде $f \in D(H), \Phi_{\vec{u}}\left(p_{1}, p_{2}\right)$ и $\Phi_{\vec{v}}\left(p_{1}, p_{2}\right)$ - функции вида (4), $\vec{u}, \vec{v} \in W^{(3)}$. Оператор $H^{*}$ действует на функиию $g\left(p_{1}, p_{2}\right)$ вида (5) по формуле

$$
\left(H^{*} g\right)\left(p_{1}, p_{2}\right)=\left(\frac{1}{2 m_{1}} p_{1}^{2}+\frac{1}{2 m_{2}} p_{2}^{2}\right) g\left(p_{1}, p_{2}\right)-u_{1}\left(p_{1}\right)-u_{2}\left(p_{2}\right)-u_{3}\left(p_{1}+p_{2}\right) .
$$

ДокАЗАТЕЛьСтво. Поскольку $H$ - неотрицательный симметрический оператор, воспользуемся теорией расширений полуограниченных симметрических операторов. Видно, что $D(H)=D(H+E), D\left(H^{*}\right)=D\left(H^{*}+E\right)$. Тогда из работы [9] следует, что область $D\left(H^{*}\right)$ разлагается в прямую сумму

$$
D\left(H^{*}\right)=D(H)+U+S_{1}^{-1} U,
$$

где $U$ - подпространство решений уравнения $\left(H^{*}+E\right) \Phi=0$, а $S_{1}$ - жесткое самосопряженное расширение оператора $H+E$. Используя разложение (5), получим, что $U$ 
состоит из функций $\Phi_{\vec{u}}\left(p_{1}, p_{2}\right)$ вида (4), где $\vec{u} \in W^{(3)}$. Нетрудно убедиться, что оператор $S_{1}^{-1}$ действует на функцию $\Phi_{\vec{u}}\left(p_{1}, p_{2}\right)$ вида (4) из $D\left(H^{*}\right)$ по формуле

$$
S_{1}^{-1} \Phi_{\vec{u}}\left(p_{1}, p_{2}\right)=\left(\frac{1}{2 m_{1}} p_{1}^{2}+\frac{1}{2 m_{2}} p_{2}^{2}+1\right)^{-1} \Phi_{\vec{u}}\left(p_{1}, p_{2}\right) .
$$

Итак, для любой функции $g\left(p_{1}, p_{2}\right) \in D\left(H^{*}\right)$ справедливо разложение (5).

Пусть $g \in D\left(H^{*}\right)$. Тогда

$$
g=f+\Phi_{\vec{u}}+S_{1}^{-1} \Phi_{\vec{v}} \quad\left(f \in D(H), \quad \Phi_{\vec{u}} \in U, \quad \Phi_{\vec{v}} \in U\right)
$$

и

$$
\begin{aligned}
H^{*} g & =H f+H^{*} \Phi_{\vec{u}}+H^{*} S_{1}^{-1} \Phi_{\vec{v}}= \\
& =H f+\left(H^{*}+E\right) \Phi_{\vec{u}}+\left(H^{*}+E\right) S_{1}^{-1} \Phi_{\vec{v}}-\Phi_{\vec{u}}-S_{1}^{-1} \Phi_{\vec{v}}= \\
& =H f+\left(\Phi_{\vec{v}}-S_{1}^{-1} \Phi_{\vec{v}}\right)-\Phi_{\vec{u}} .
\end{aligned}
$$

Отсюда следует, что

$$
\begin{aligned}
\left(H^{*} g\right)\left(p_{1}, p_{2}\right)= & \left(\frac{1}{2 m_{1}} p_{1}^{2}+\frac{1}{2 m_{2}} p_{2}^{2}\right)\left[f\left(p_{1}, p_{2}\right)+\Phi_{\vec{u}}\left(p_{1}, p_{2}\right)+S_{1}^{-1} \Phi_{\vec{v}}\left(p_{1}, p_{2}\right)\right]- \\
& -\left(\frac{1}{2 m_{1}} p_{1}^{2}+\frac{1}{2 m_{2}} p_{2}^{2}+1\right)^{-1} \Phi_{\vec{u}}\left(p_{1}, p_{2}\right)= \\
= & \left(\frac{1}{2 m_{1}} p_{1}^{2}+\frac{1}{2 m_{2}} p_{2}^{2}\right) g\left(p_{1}, p_{2}\right)-u_{1}\left(p_{1}\right)-u_{2}\left(p_{2}\right)-u_{3}\left(p_{1}+p_{2}\right) .
\end{aligned}
$$

Лемма доказана.

Пусть $A$-произвольный линейный оператор с плотной в $W^{(3)}$ областью определения $D(A)$, а $H_{A}$ - сужение оператора $H^{*}$ на область

$$
D\left(H_{A}\right)=\left\{g \in D\left(H^{*}\right): \quad g=f+\Phi_{\vec{u}}+S_{1}^{-1} \Phi_{A \vec{u}}, \quad f \in D(H), \quad \vec{u} \in D(A)\right\},
$$

т.е. оператор $H_{A}$ получается сужением оператора $H^{*}$ на множество функций $g\left(p_{1}, p_{2}\right)$, для которых в разложении (5) выполняется условие вида

$$
\vec{v}=A \vec{u} \quad(\vec{u} \in D(A)) .
$$

Возьмем два произвольных элемента $g=f+\Phi_{\vec{u}}+S_{1}^{-1} \Phi_{A \vec{u}}$ и $g^{\prime}=f^{\prime}+\Phi_{\vec{u}^{\prime}}+S_{1}^{-1} \Phi_{A \vec{u}^{\prime}}$ из $D\left(H_{A}\right)$. Для них справедливо следуюшее соотношение:

$$
\left(H_{A} g, g^{\prime}\right)=\left(g, H_{A} g^{\prime}\right)+\left(\Phi_{A \vec{u}}, \Phi_{\vec{u}^{\prime}}\right)-\left(\Phi_{\vec{u}}, \Phi_{A \vec{u}^{\prime}}\right),
$$

где $(\cdot, \cdot)$ - скалярное произведение в $L_{2}$. Так как пространства $\Re_{-1}(H)$ и $W^{(3)}$ изоморфны, а также выполняются равенства $\left(\Phi_{A \vec{u}}, \Phi_{\vec{u}^{\prime}}\right)=\left[A \vec{u}, \vec{u}^{\prime}\right]$ и $\left(\Phi_{\vec{u}}, \Phi_{A \vec{u}^{\prime}}\right)=\left[\vec{u}, A \vec{u}^{\prime}\right]$, где $[\cdot, \cdot \cdot]$ - скалярное произведение в $W^{(3)}$, то соотношение (8) можно переписать в виде

$$
\left(H_{A} g, g^{\prime}\right)=\left(g, H_{A} g^{\prime}\right)+\left[A \vec{u}, \vec{u}^{\prime}\right]-\left[\vec{u}, A \vec{u}^{\prime}\right] .
$$

Из равенства (9) следует, что оператор $H_{A}$ является симметрическим оператором в $L_{2}$ тогда и только тогда, когда $A$ является симметрическим оператором в $W^{(3)}$. 
Лемма 2. Расширение $H_{A}$ оператора $H$, задаваемое с помощью условия (7), является самосопряженным оператором тогда и только тогда, когда А самосопрязсенный оператор в $W^{(3)}$.

ДокаЗАТЕЛЬство. Сначала докажем, что если $A$ - симметрический оператор в $W^{(3)}, A^{*}$ - сопряженный к $A$ оператор, то $H_{A}^{*}=H_{A^{*}}$, где $H_{A}^{*}$ - сопряженньй к $H_{A}$ оператор.

Пусть $\psi$ - произвольный элемент из $D\left(H_{A^{*}}\right)$. Тогда сушествуют элементы $f_{\psi} \in$ $D(H), \vec{v} \in D\left(A^{*}\right)$ такие, что $\psi=f_{\psi}+\Phi_{\vec{v}}+S_{1}^{-1} \Phi_{A^{*} \vec{v}}$ и для любого $g=f_{g}+\Phi_{\vec{u}}+S_{1}^{-1} \Phi_{A \vec{u}}$ из $D\left(H_{A}\right)$ выполняется равенство

$$
\left(H_{A} g, \psi\right)=\left(g, H_{A^{*}} \psi\right)+[A \vec{u}, \vec{v}]-\left[\vec{u}, A^{*} \vec{v}\right]
$$

или в силу того, что $[A \vec{u}, \vec{v}]=\left[\vec{u}, A^{*} \vec{v}\right]$, выполняются равенства

$$
\left(H_{A} g, \psi\right)=\left(g, H_{A^{*}} \psi\right)=\left(g, H_{A}^{*} \psi\right)
$$

Итак, если $\psi \in D\left(H_{A^{*}}\right)$, то $\psi \in D\left(H_{A}^{*}\right)$ и $H_{A}^{*} \psi=H_{A^{*}} \psi$, т.е. $H_{A^{*}} \subseteq H_{A}^{*}$.

Пусть теперь $\widetilde{\psi}$ - произвольный элемент из $D\left(H_{A}^{*}\right)$. Тогда сушествуют элементы $f_{\widetilde{\psi}} \in D(H), \vec{w}, \vec{w}^{*} \in W^{(3)}$ такие, что $\widetilde{\psi}=f_{\widetilde{\psi}}+\Phi_{\vec{w}}+S_{1}^{-1} \Phi_{\vec{w}^{*}}$ и для любого $g=f_{g}+$ $\Phi_{\vec{u}}+S_{1}^{-1} \Phi_{A \vec{u}}$ из $D\left(H_{A}\right)$ выполняется равенство

$$
\left(H_{A} g, \widetilde{\psi}\right)=\left(g, H_{A}^{*} \widetilde{\psi}\right)+[A \vec{u}, \vec{w}]-\left[\vec{u}, \vec{w}^{*}\right]
$$

Так как $\left(H_{A} g, \widetilde{\psi}\right)=\left(g, H_{A}^{*} \widetilde{\psi}\right)$, то для любого $\vec{u} \in D(A)$ получаем равенство $[A \vec{u}, \vec{w}]=$ $\left[\vec{u}, \vec{w}^{*}\right]$. Это означает, что $\vec{w} \in D\left(A^{*}\right)$ и $\vec{w}^{*}=A^{*} \vec{w}$. Отсюда следует, что $\widetilde{\psi}=f_{\widetilde{\psi}}+$ $\Phi_{\vec{w}}+S_{1}^{-1} \Phi_{A^{*}} \underset{w}{w}$. В силу произвольности элемента $\widetilde{\psi}$ получим, что $D\left(H_{A}^{*}\right) \subseteq D\left(H_{A^{*}}\right)$ и $H_{A}^{*} \widetilde{\psi}=H_{A^{*}} \widetilde{\psi}$. Таким образом, доказано равенство $H_{A}^{*}=H_{A^{*}}$. Утверждение леммы легко выводится из последнего равенства.

\section{3. ВЫБОР ОПЕРАTOPA $A$}

Для тех элементов $g \in D\left(H^{*}\right)$, которым соответствуют достаточно быстро убывающие функции $u_{1}, u_{2}, u_{3}$ в разложении $(5)$, определены асимптотики

$$
\begin{array}{rlrl}
\int_{\left|p^{\prime}\right|<N} g\left(p, p^{\prime}\right) d p^{\prime} & =4 \pi \cdot 2 m_{j} u_{k}(p) N+B_{k}(p)+o(1) & \text { при } \quad & N \rightarrow \infty, \\
\int_{\Gamma_{p} \cap\left|\frac{m_{1}}{M} p_{2}-\frac{m_{2}}{M} p_{1}\right|<N} g\left(p_{1}, p_{2}\right) d v_{p} & =4 \pi \eta u_{3}(p) N+B_{3}(p)+o(1) & \text { при } \quad N \rightarrow \infty,
\end{array}
$$


где $j, k=1,2, j \neq k$,

$$
\begin{aligned}
B_{k}(p)= & -2 \pi^{2}\left(2 m_{j}\right)^{\frac{3}{2}} \sqrt{\frac{1}{2 m_{k}} p^{2}+1} u_{k}(p)+\pi^{2}\left(2 m_{j}\right)^{\frac{3}{2}}\left(\frac{1}{2 m_{k}} p^{2}+1\right)^{-\frac{1}{2}} v_{k}(p)+ \\
& +\int_{\mathbb{R}^{3}} \frac{u_{j}(q)+u_{3}(p+q)}{\left(2 m_{k}\right)^{-1} p^{2}+\left(2 m_{j}\right)^{-1} q^{2}+1} d q+ \\
& +\int_{\mathbb{R}^{3}} \frac{v_{j}(q)+v_{3}(p+q)}{\left(\left(2 m_{k}\right)^{-1} p^{2}+\left(2 m_{j}\right)^{-1} q^{2}+1\right)^{2}} d q, \\
B_{3}(p)= & -2 \pi^{2} \eta^{\frac{3}{2}} \sqrt{\frac{1}{2 M} p^{2}+1} u_{3}(p)+\pi^{2} \eta^{\frac{3}{2}}\left(\frac{1}{2 M} p^{2}+1\right)^{-\frac{1}{2}} v_{3}(p)+ \\
& +\int_{\Gamma_{p}} \frac{u_{1}\left(p_{1}\right)+u_{2}\left(p_{2}\right)}{\left(2 m_{1}\right)^{-1} p_{1}^{2}+\left(2 m_{1}\right)^{-1} p_{2}^{2}+1} d v_{p}+ \\
& +\int_{\Gamma_{p}} \frac{v_{1}\left(p_{1}\right)+v_{2}\left(p_{2}\right)}{\left(\left(2 m_{1}\right)^{-1} p_{1}^{2}+\left(2 m_{2}\right)^{-1} p_{2}^{2}+1\right)^{2}} d v_{p} .
\end{aligned}
$$

Обозначим через $H_{\varepsilon}^{*}, \varepsilon=\left(\varepsilon_{1}, \varepsilon_{2}, \varepsilon_{3}\right) \in \mathbb{R}^{3}$, сужение оператора $H^{*}$ на область $D\left(H_{\varepsilon}^{*}\right)$ функций $g\left(p_{1}, p_{2}\right)$ из $D\left(H^{*}\right)$, удовлетворяюших условиям

$$
\begin{aligned}
& B_{k}(p)=2 \pi^{2}\left(2 m_{j}\right)^{\frac{3}{2}} \varepsilon_{k} u_{k}(p), \quad j, k=1,2, \quad j \neq k, \\
& B_{3}(p)=2 \pi^{2} \eta^{\frac{3}{2}} \varepsilon_{3} u_{3}(p) .
\end{aligned}
$$

Рассмотрим семейство операторов $\{T(z)\}, z \in \mathbb{C} \backslash[0, \infty)$, в $L_{2}^{(3)}$, действуюших по формулам

$$
\begin{gathered}
(T(z) \vec{u})_{k}(p)=2 \pi^{2}\left(2 m_{j}\right)^{\frac{3}{2}} \sqrt{\frac{1}{2 m_{j}} p^{2}-z} u_{k}(p)-\int_{\mathbb{R}^{3}} \frac{u_{j}(q)+u_{3}(p+q)}{\left(2 m_{k}\right)^{-1} p^{2}+\left(2 m_{j}\right)^{-1} q^{2}-z} d q, \\
j, k=1,2, \quad j \neq k, \\
(T(z) \vec{u})_{3}(p)=2 \pi^{2} \eta^{\frac{3}{2}} \sqrt{\frac{1}{2 M} p^{2}-z} u_{3}(p)-\int_{\Gamma_{p}} \frac{u_{1}\left(p_{1}\right)+u_{2}\left(p_{2}\right)}{\left(2 m_{1}\right)^{-1} p_{1}^{2}+\left(2 m_{2}\right)^{-1} p_{2}^{2}-z} d v_{p}
\end{gathered}
$$

на множестве $D$ троек функций $\vec{u}(p)=\left\{u_{k}(p)\right\} \in L_{2}^{(3)}$ таких, что

$$
\left|u_{k}(p)\right| \leqslant \frac{\text { const }}{1+|p|^{3}}, \quad k=1,2,3 .
$$

Введем в рассмотрение семейство операторов в $W^{(3)}$

$$
A_{\varepsilon}=K^{-1}\left[T(-1)+2 \pi^{2} E\right],
$$

которые также определены на множестве $D$. Здесь

$$
\begin{aligned}
& (E \vec{u})_{1}(p)=\left(2 m_{2}\right)^{\frac{3}{2}} \varepsilon_{1} u_{1}(p), \\
& (E \vec{u})_{2}(p)=\left(2 m_{1}\right)^{\frac{3}{2}} \varepsilon_{2} u_{2}(p), \\
& (E \vec{u})_{3}(p)=\eta^{\frac{3}{2}} \varepsilon_{3} u_{3}(p),
\end{aligned}
$$

$\varepsilon=\left(\varepsilon_{1}, \varepsilon_{2}, \varepsilon_{3}\right)-$ произвольная тройка вешественных чисел. 
Лемма 3. Для любой тройки $\varepsilon=\left(\varepsilon_{1}, \varepsilon_{2}, \varepsilon_{3}\right) \in \mathbb{R}^{3}$ оператор $A_{\varepsilon}^{*}$, сопряжсенньй $\kappa$ оператору $A_{\varepsilon}$, определяется по формулам

$$
A_{\varepsilon}^{*}=K^{-1}\left[T^{*}(-1)+2 \pi^{2} E\right], \quad D\left(A_{\varepsilon}^{*}\right)=D^{*},
$$

где $T^{*}(-1)$ - оператор, сопряженныи $к$ оператору $T(-1), D^{*}=D\left(T^{*}(-1)\right)$ - его область определения.

ДокАЗАТЕльство. Пусть $\vec{v}$ - произвольный элемент из $D\left(A_{\varepsilon}^{*}\right)$. Тогда из $(13)$ следует, что для всех $\vec{u} \in D$ справедливы равенства

$$
\left[A_{\varepsilon} \vec{u}, \vec{v}\right]=\left(\left[T(-1)+2 \pi^{2} E\right] \vec{u}, \vec{v}\right), \quad\left[A_{\varepsilon} \vec{u}, \vec{v}\right]=\left(\vec{u}, K A_{\varepsilon}^{*} \vec{v}\right),
$$

откуда в свою очередь следует, что $\vec{v} \in D^{*}$ и $A_{\varepsilon}^{*} \vec{v}=K^{-1}\left[T^{*}(-1)+2 \pi^{2} E\right] \vec{v}$. В силу произвольности $\vec{v}$ получим, что $D\left(A_{\varepsilon}^{*}\right) \subset D^{*}$.

Пусть теперь $\vec{w}$ - произвольньй элемент из $D^{*}$. Тогда для всех $\vec{u}$ из $D$ справедливы равенства

$$
\begin{aligned}
{\left[A_{\varepsilon} \vec{u}, \vec{w}\right] } & =\left(\left[T(-1)+2 \pi^{2} E\right] \vec{u}, \vec{w}\right)=\left(\vec{u},\left[T^{*}(-1)+2 E\right] \vec{w}\right)= \\
& =\left(K \vec{u}, K^{-1}\left[T^{*}(-1)+2 \pi^{2} E\right] \vec{w}\right)=\left[\vec{u}, K^{-1}\left(T^{*}(-1)+2 \pi^{2} E\right) \vec{w}\right] .
\end{aligned}
$$

Это означает, что $\vec{w} \in D\left(A_{\varepsilon}^{*}\right)$ и $A_{\varepsilon}^{*} \vec{w}=K^{-1}\left[T^{*}(-1)+2 \pi^{2} E\right] \vec{w}$. Таким образом, $A_{\varepsilon}^{*}=$ $K^{-1}\left[T^{*}(-1)+2 \pi^{2} E\right]$ и $D\left(A_{\varepsilon}^{*}\right)=D^{*}$.

Из формул (10) и условий (11) видно, что если в формуле (7) оператор $A$ заменить на оператор $A_{\varepsilon}^{*}$, то полученное сужение $H_{A_{\varepsilon}^{*}}$ оператора $H^{*}$ совпадает с оператором $H_{\varepsilon}^{*}$, т.е. $H_{\varepsilon}^{*}+H_{A_{\varepsilon}^{*}}$. Поскольку $H_{\varepsilon}^{*}=H_{A_{\varepsilon}^{*}}=\left(H_{A_{\varepsilon}^{*}}\right)^{*}$, то оператор $H_{\varepsilon}$ совпадает с оператором $H_{A_{\varepsilon}}$. Таким образом, симметрическое расширение $H_{\varepsilon}$ оператора $H$ получается сужением оператора $H^{*}$ на область $D\left(H_{A_{\varepsilon}}\right)=D\left(H_{\varepsilon}\right)$ с помощью условия $\vec{v}=A_{\varepsilon} \vec{u}$.

Применим выписанные соотношения для построения резольвенты оператора $H_{\varepsilon}$. Полагая $g=\left(H_{\varepsilon}-z E\right)^{-1} h$, из уравнения

$$
\left(H_{\varepsilon}-z E\right) g=h, \quad \operatorname{Im} z \neq 0,
$$

и формулы (6) находим, что для любого $h \in L_{2}$ функция $g$ имеет вид

$$
g\left(p_{1}, p_{2}\right)=\frac{h\left(p_{1}, p_{2}\right)+u_{1}\left(p_{1}\right)+u_{2}\left(p_{2}\right)+u_{3}\left(p_{1}+p_{2}\right)}{\left(2 m_{1}\right)^{-1} p_{1}^{2}+\left(2 m_{2}\right)^{-1} p_{2}^{2}-z},
$$

где $u_{1}, u_{2}, u_{3}$ - неизвестные функции.

Сравнивая (14) с (5) и используя условие $\vec{v}=A_{\varepsilon} \vec{u}$, мы приходим к системе уравнений для определения функций $u_{1}, u_{2}, u_{3}$ через $h\left(p_{1}, p_{2}\right)$ :

$$
\begin{array}{r}
2 \pi^{2}\left(2 m_{j}\right)^{\frac{3}{2}}\left(\sqrt{\frac{1}{2 m_{k}} p^{2}-z}+\varepsilon_{k}\right) u_{k}(p)-\int_{\mathbb{R}^{3}} \frac{u_{j}(q)+u_{3}(p+q)}{\left(2 m_{k}\right)^{-1} p^{2}+\left(2 m_{j}\right)^{-1} q^{2}-z} d q=\chi_{k}(p ; z), \\
2 \pi^{2} \eta^{\frac{3}{2}}\left(\sqrt{\frac{1}{2 M} p^{2}-z}+\varepsilon_{3}\right) u_{3}(p)-\int_{\Gamma_{p}} \frac{u_{1}\left(p_{1}\right)+u_{2}\left(p_{2}\right)}{\left(2 m_{1}\right)^{-1} p_{1}^{2}+\left(2 m_{2}\right)^{-1} p_{2}^{2}-z} d v_{p}=\chi_{3}(p ; z),
\end{array}
$$


где $j, k=1,2, j \neq k$,

$$
\begin{aligned}
\chi_{k}\left(p_{k} ; z\right) & \equiv \int_{\mathbb{R}^{3}} \frac{h\left(p_{1}, p_{2}\right)}{\left(2 m_{1}\right)^{-1} p_{1}^{2}+\left(2 m_{2}\right)^{-1} p_{2}^{2}-z} d p_{j}, \\
\chi_{3}(p ; z) & \equiv \int_{\Gamma_{p}} \frac{h(p, q)}{\left(2 m_{1}\right)^{-1} p^{2}+\left(2 m_{2}\right)^{-1} q^{2}-z} d v_{q} .
\end{aligned}
$$

Заметим, что из лемм 2 и 3 и из соотношений (5), (7) и (13) легко следует, что самосопряженность $H_{\varepsilon}$ (для всех $\varepsilon \in \mathbb{R}^{3}$ ) равносильна существенной самосопряженности оператора $T(-1)$, причем индексы дефекта $T(-1)$ совпадают с индексами дефекта оператора $H_{\varepsilon}$.

\section{4. ОСНОВНЫЕ РЕЗУЛЬТАТЫ}

Teорема 1. Оператор $T(-1)$ имеет ненулевые, равные и конечные индексы дефекта.

ДоКАЗАТЕЛЬСТво. Пусть $T$ - симметрический оператор, определенньй на области $D$ (см. (12)) и действуюший по формулам

$$
\begin{aligned}
&(T \vec{u})_{k}(p)=4 \pi^{2} m_{j} \sqrt{\frac{m_{j}}{m_{k}}}|p| u_{k}(p)-\int_{\mathbb{R}^{3}} \frac{u_{j}(q)+u_{3}(p+q)}{\left(2 m_{k}\right)^{-1} p^{2}+\left(2 m_{j}\right)^{-1} p^{2}} d q, \\
& j, k=1,2, \quad j \neq k, \\
&(T \vec{u})_{3}(p)=2 \pi^{2} \eta \sqrt{\frac{\eta}{2 M}}|p| u_{3}(p)-\int_{\Gamma_{p}} \frac{u_{1}\left(p_{1}\right)+u_{2}\left(p_{2}\right)}{\left(2 m_{1}\right)^{-1} p_{1}^{2}+\left(2 m_{2}\right)^{-1} p^{2}} d v_{p} .
\end{aligned}
$$

Так как прибавление ограниченного самосопряженного оператора не изменяет индексов дефекта и $T(-1)-T$ является компактным самосопряженным оператором, то вместо $T(-1)$ достаточно рассмотреть оператор $T$.

Заметим, что оператор $T$ коммутирует с операторами представления группы врашений $O_{3}$ в $L_{2}^{(3)}$ :

$$
\left(V_{g} \vec{u}\right)_{k}(p)=u_{k}\left(g^{-1} p\right), \quad \vec{u} \in L_{2}^{(3)}, \quad g \in O_{3},
$$

и приводится каждым подпространством $L_{l}$ троек вида

$$
u_{k}^{l}(p)=\sum_{m=-1}^{l} u_{k}^{m, l}(|p|) Y_{m}^{l}(\theta, \varphi), \quad k=1,2,3,
$$

где $|p|=r, \theta, \varphi$ - сферические координаты $p, Y_{m}^{l}(\theta, \varphi)$ - сферические функции веса $l$ (см. [10]), $u_{k}^{m, l}$ - функции, зависящие от $|p|$. Часть оператора $T$, действуюшую в $L_{l}$, обозначим через $T_{l}$. Рассмотрим сначала оператор $T_{0}$, определенный на тройках сферически-симметричных функций $\left\{u_{k}(p)\right\}=\left\{u_{k}(|p|)\right\}$. Выполняя в формулах (15) для сферически-симметричных функций интегрирования по углам, убедимся в том, 
что оператор $T_{0}$ унитарно-эквивалентен оператору $S_{0}$, действуюшему в пространстве $L_{2}^{(3)}\left(R_{+}, r^{2} d r\right) \equiv L_{2}\left(R_{+}, r^{2} d r\right) \oplus L_{2}\left(R_{+}, r^{2} d r\right) \oplus L_{2}\left(R_{+}, r^{2} d r\right)$ по формулам

$$
\begin{aligned}
\left(S_{0} \vec{u}\right)_{k}(r)= & 4 \pi^{2} m_{j} \sqrt{\frac{m_{j}}{m_{k}}} r u_{k}(r)-4 \pi \int_{0}^{+\infty} \frac{t^{2} u_{j}(t)}{\left(2 m_{k}\right)^{-1} r^{2}+\left(2 m_{j}\right)^{-1} t^{2}} d t- \\
& -2 \pi m_{j} \int_{0}^{\infty} \frac{t}{r} \ln \left[\frac{\eta^{-1} r^{2}+m_{j}^{-1} r t+\left(2 m_{j}\right)^{-1} t^{2}}{\eta^{-1} r^{2}-m_{j}^{-1} r t+\left(2 m_{j}\right)^{-1} t^{2}}\right] u_{3}(t) d t, \\
& j, k=1,2, \quad j \neq k, \\
\left(S_{0} \vec{u}\right)_{3}(r)= & 2 \pi^{2} \sqrt{\frac{\eta^{3}}{2 M}} r u_{3}(r)- \\
& -2 \pi \sum_{k \neq j} m_{j} \int_{0}^{+\infty} \frac{t}{r} \ln \left[\frac{\left(2 m_{j}\right)^{-1} r^{2}+m_{j}^{-1} r t+\eta^{-1} t^{2}}{\left(2 m_{j}\right)^{-1} r^{2}-m_{j}^{-1} r t+\eta^{-1} t^{2}}\right] u_{k}(t) d t .
\end{aligned}
$$

Область определения $D\left(S_{0}\right)$ оператора $S_{0}$ состоит из троек функций $\left\{u_{k}(r)\right\} \in$ $L_{2}^{(3)}\left(R_{+}, r^{2} d r\right)$ таких, что $u_{k}(r)$ удовлетворяют условию $(12)$.

После унитарного в $L_{2}^{(3)}\left(R_{+}, r^{2} d r\right)$ преобразования Меллина

$$
v_{j}(s)=\int_{0}^{+\infty} r^{-i s+\frac{1}{2}} u_{j}(r) d r
$$

где $\vec{u}(r)=\left\{u_{j}(r)\right\} \in D\left(S_{0}\right)$, оператор $S_{0}$ перейдет в оператор $\widehat{S}_{0}$ :

$$
\left(\widehat{S}_{0} \vec{v}\right)_{j}(s)=\left(F\left(s+\frac{i}{2}\right) \vec{v}(s+i)\right)_{j}
$$

$(F(s)$ - некоторая матрица, описанная ниже, $j=1,2,3)$, действующий на области $D\left(\widehat{S}_{0}\right)$ троек $\left\{v_{j}(s)\right\}$ таких, что функции $v_{j}(s)$ аналитичны в полосе $0 \leqslant \operatorname{Im} s \leqslant 1$ и справедливы неравенства

$$
\int_{-\infty}^{+\infty}\left|v_{j}(x+i \alpha)\right|^{2} d x<\infty, \quad 0 \leqslant \alpha \leqslant 1, \quad j=1,2,3 .
$$

Здесь матрица $F(s)$ действует на тройку $\vec{v}(s)=\left\{v_{j}(s)\right\}_{1 \leqslant j \leqslant 3}$ по формулам

$$
\begin{aligned}
(F(s) \vec{v})_{j}(s)= & 4 \pi^{2} m_{k} \sqrt{\frac{m_{k}}{m_{j}}} v_{j}(s)-4 \pi^{2} m_{k}\left(\frac{M}{m_{j}}\right)^{\frac{i s}{2}} \frac{\operatorname{sh}\left(\varphi_{j} s\right)}{s \operatorname{ch}(\pi s / 2)} v_{3}(s)- \\
& -4 \pi^{2} \sqrt{m_{1} m_{2}}\left(\frac{m_{k}}{m_{j}}\right)^{\frac{i s}{2}} \frac{v_{k}(s)}{\operatorname{ch}(\pi s / 2)}, \quad j, k=1,2, \quad j \neq k, \\
(F(s) \vec{v})_{3}(s)= & 2 \pi^{2} \sqrt{\frac{\eta^{3}}{2 M}} v_{3}(s)-4 \pi^{2} \sum_{j \neq k} m_{k}\left(\frac{m_{j}}{M}\right)^{\frac{i s}{2}} \frac{\operatorname{sh}\left(\varphi_{j} s\right)}{s \operatorname{ch}(\pi s / 2)} v_{j}(s),
\end{aligned}
$$

где углы $\varphi_{1}$ и $\varphi_{2}$ определяются из соотношений

$$
\sin \varphi_{j}=\sqrt{\frac{m_{j}}{M}}, \quad 0<\varphi_{j}<\frac{\pi}{2}, \quad j=1,2
$$


Повторяя рассуждения из работы [8], получим, что область определения $D\left(\widehat{S}_{0}^{*}\right)$ сопряженного оператора $\widehat{S}_{0}^{*}$ состоит из троек функций вида $\vec{w}(s)=[F(s-i / 2)]^{-1} \vec{v}(s)$, где $\vec{v}(s) \in D\left(\widehat{S}_{0}\right)$ (это легко выводится из теоремы братьев Рисс о мерах на окружности, ортогональных степеням (см. [11])).

Лемма 4. Функиия $\operatorname{det} F(s), s \in \mathbb{R}$, обращается в нуль только в двух точках $s= \pm s_{0}$, причем $\operatorname{rank} F\left( \pm s_{0}\right)=2$.

ДокаЗАТЕЛЬство. Заметим, что

$$
\operatorname{det} F(s)=64 \pi^{6} \frac{\left(m_{1} m_{2}\right)^{\frac{5}{2}}}{M^{2}}\left[1-\lambda_{1}^{2}(s)-\lambda_{2}^{2}(s)-\lambda_{3}^{2}(s)-2 \lambda_{1}(s) \lambda_{2}(s) \lambda_{3}(s)\right]
$$

где

$$
\lambda_{j}(s)=\frac{M}{\sqrt{m_{1} m_{2}}} \frac{\operatorname{sh}\left(\varphi_{j} s\right)}{s \operatorname{ch}(\pi s / 2)}, \quad j=1,2, \quad \lambda_{3}(s)=\frac{1}{\operatorname{ch}(\pi s / 2)} .
$$

Поскольку $\lambda_{j}(s)$ - четные функции, достаточно рассмотреть случай $s \geqslant 0$. Из условия $0<\varphi<\pi / 2$ (см. (18)) следует, что функции $\lambda_{j}(s), j=1,2,3$, монотонно убывают и $\lambda_{j}(s) \rightarrow 0$ при $s \rightarrow+\infty$. Следовательно, на отрезке $[0,+\infty)$ функция $\operatorname{det} F(s)$ монотонно возрастает и стремится к $64 \pi^{6}\left(m_{1} m_{2}\right)^{5 / 2} / M^{2}$ при $s \rightarrow+\infty$, но

$$
\operatorname{det} F(0)=-64 \pi^{6}\left(m_{1} m_{2}\right)^{\frac{3}{2}}\left(\varphi_{1}+\varphi_{2}\right)^{2}<0 .
$$

Таким образом, сушествует единственная точка $s_{0}>0$ такая, что $\operatorname{det} F\left(s_{0}\right)=0$.

Из гладкости и строгой монотонности функции $\operatorname{det} F(s)$ на отрезках $(-\infty, 0]$ и $[0,+\infty)$ следует, что

$$
\left.\frac{d}{d s}(\operatorname{det} F(s))\right|_{s= \pm s_{0}} \neq 0 \text {. }
$$

Пусть функции $k_{j}(s), j=1,2,3,-$ собственные значения матрицы $F(s)$. Поскольку $\operatorname{det} F(s)=k_{1}(s) \cdot k_{2}(s) \cdot k_{3}(s)$, то функции $k_{j}(s)$ являются гладкими. Тогда из условия (19) следует, что в точках $s= \pm s_{0}$ в нуль обращается только какая-то одна из функций $k_{j}(s)$. Лемма доказана.

Вернемся к доказательству теоремы. Из леммы 4 следует, что $D\left(\widehat{S}_{0}^{*}\right)$ кроме элементов $\widehat{S}_{0}$ включает в себя тройки функций $\vec{e}^{(+)}\left(s-s_{0}-i / 2\right)^{(-1)}, \vec{e}^{(-)}\left(s+s_{0}-i / 2\right)^{(-1)}$, где $\vec{e}^{( \pm)}=\left\{\vec{e}_{j}^{( \pm)}\right\}-$решения уравнений

$$
F\left( \pm s_{0}\right) \vec{e}^{( \pm)}=0
$$

Это означает, что область $D\left(\widehat{S}_{0}^{*}\right)$ состоит из элементов вида $\vec{q}(s)=\left\{q_{j}(s)\right\}$,

$$
q_{j}(s)=\frac{c_{1} e_{j}^{-}}{s+s_{0}-i / 2}+\frac{c_{2} e_{j}^{+}}{s-s_{0}-i / 2}+g_{j}(s), \quad j=1,2,3,
$$

где $\vec{g}(s)=\left\{g_{j}(s)\right\} \in D\left(\widehat{S}_{0}\right)$. Таким образом, область $D\left(\widehat{S}_{0}\right)$ имеет в $D\left(\widehat{S}_{0}^{*}\right)$ коразмерность 2. Отсюда следует, что индексы дефекта оператора $\widehat{S}_{0}$ (и оператора $S_{0}$ ) равны либо $(2,0)$, либо $(0,2)$, либо $(1,1)$. Однако в силу вешественности оператора $S_{0}$ 
его индексы дефекта одинаковы и равны $(1,1)$. Поскольку операторы $T_{0}$ и $S_{0}$ унитарно-эквивалентны, то их индексы дефекта одинаковые. Следовательно, оператор $T_{0}$ имеет индексы дефекта $(1,1)$.

Теперь рассмотрим случай $l \geqslant 1$. Как и в случае $l=0$, можно показать, что индексы дефекта оператора $T_{l}(-1)$ равны и не превышают $(1,1)$. Кроме того, для любых $m_{1}$ и $m_{2}$ сушествует натуральное число $l_{1}=l\left(m_{1}, m_{2}\right)$ такое, что при $l \geqslant l_{1}$ индексы дефекта операторов $T_{l}(-1)$ равны $(0,0)$. Отсюда следует, что оператор $T(-1)$ имеет равные и конечные индексы дефекта $(n, n)$, где $1 \leqslant n \leqslant l_{1}$.

В доказательстве теоремы 1 фактически были описаны дефектные векторы операторов $T_{0}$ и $T_{0}(-1)$. Применив обшую теорию Неймана самосопряженных расширений симметрических операторов (см. [2]), придем к семейству $\left\{T_{0 \beta}(-1)\right\}, \beta \in \mathbb{C},|\beta|=1$, самосопряженных расширений оператора $T_{0}(-1)$. Описанию области определения оператора $T_{0 \beta}(-1)$ посвяшается следуюшая теорема.

Tеорема 2. Область определения оператора $T_{0 \beta}(-1)$ состоит из әлементов вида $\vec{v}(p)=\vec{v}(|p|)=\left\{v_{j}(p)\right\}$, əде

$$
v_{j}(p)=v_{j}(r)=c \frac{e_{j} r^{i s_{0}}+\beta \bar{e}_{j} r^{-i s_{0}}}{r^{2}+1}+u_{j}(r), \quad j=1,2,3 .
$$

Здесь $r=|p| \in R_{+}, \vec{u}(p)=\left\{u_{j}(p)\right\} \in D\left(T_{0}(-1)\right),\left\{e_{j}\right\}-$ вектор, введенныц в в доказательстве теоремы 1 (см. (20)), $\beta \in \mathbb{C},|\beta|=1$ - параметр самосопряженного расиирения $T_{0 \beta}(-1)$ оператора $T_{0}(-1)$.

ДокАЗАТЕЛЬСТво. Из теоремы 1 следует, что дефектное пространство

$$
\Re_{z}=\left(\operatorname{Ran}\left(T_{0}(-1)-z I\right)\right)^{\perp}
$$

оператора $T_{0}(-1)$ одномерно, а из соотношения (21) видно, что дефектный вектор $\vec{\theta}^{z}(r)=\left\{\theta_{j}^{z}(r)\right\} \in \Re_{z}$ оператора $T_{0}(-1)$ имеет вид

$$
\theta_{j}^{z}(r)=\frac{e_{j} r^{i s_{0}}+\zeta \bar{e}_{j} r^{-i s_{0}}}{r^{2}+1}+w_{j}(r), \quad j=1,2,3,
$$

для некоторого комплексного числа $\zeta$ и элемента $\vec{w}(r)=\left\{w_{j}(r)\right\} \in D\left(T_{0}(-1)\right)$. Следовательно,

$$
\theta_{j}^{\bar{z}}(r)=\overline{\theta_{j}^{z}(r)} \frac{\bar{\zeta} e_{j} r^{i s_{0}}+\bar{e}_{j} r^{-i s_{0}}}{r^{2}+1}+\bar{w}_{j}(r)
$$

с точностью до умножения на константу.

Рассмотрим произвольное самосопряженное расширение $\widehat{T}_{0}(-1)$ оператора $T_{0}(-1)$. Поскольку $\left\|\theta^{\bar{z}}\right\|=\left\|\theta^{z}\right\|$, из формул Неймана (см. [2]) следует, что область определения $D\left(\widehat{T}_{0}(-1)\right)$ оператора $\widehat{T}_{0}(-1)$ состоит из элементов вида $\vec{v}(p)=\vec{v}(|p|)=\left\{v_{j}(p)\right\}$, $v_{j}(p)=\theta_{j}^{z}(p)+\widehat{\beta} \theta_{j}^{\bar{z}}(p)+u_{j}(p)$, где $\left\{u_{j}(p)\right\} \in D\left(T_{0}(-1)\right),\left\{\theta_{j}^{z}(p)\right\} \in \Re_{z}, j=1,2,3$, для 
некоторой константы $\widehat{\beta} \in \mathbb{C},|\widehat{\beta}|=1$, характеризуюшей расширение $\widehat{T}_{0}(-1)$. Таким образом, с точностью до умножения на константу элемент из $D\left(\widehat{T}_{0}(-1)\right)$ будет иметь вид $\vec{v}(p)=\left\{v_{j}(p)\right\}$,

$$
v_{j}(r)=\frac{e_{j} r^{i s_{0}}+\frac{\zeta+\widehat{\beta}}{1+\bar{\zeta} \widehat{\beta}} \bar{e}_{j} r^{-i s_{0}}}{r^{2}+1}+u_{j}(r), \quad j=1,2,3,
$$

$\left\{u_{j}(r)\right\}=\left\{u_{j}(|p|)\right\}=\left\{u_{j}(p)\right\} \in D\left(T_{0}(-1)\right)$. Легко заметить, что если обозначить $\beta=$ $(\zeta+\widehat{\beta}) /(1+\bar{\zeta} \widehat{\beta})$, то $|\beta|=1$ для $\zeta \in C^{1}, \widehat{\beta} \in C^{1},|\widehat{\beta}|=1$. Но это влечет утверждение теоремы (если принять обозначение $\left.\widehat{T}_{0}(-1)=T_{0 \beta}(-1)\right)$.

Видно, что оператор $H_{\varepsilon}$ коммутирует с операторами группы врашений $O_{3}$ в $L_{2} \equiv$ $L_{2}\left((\mathbb{R})^{3}\right)^{2}$ :

$$
\left(V_{g}^{(2)} \psi\right)\left(p_{1}, p_{2}\right)=\psi\left(g^{-1} p_{1}, g^{-1} p_{2}\right), \quad \psi \in L_{2}, \quad g \in O_{3},
$$

и, следовательно, он приводится каждым подпространством $\aleph_{l}(l=0,1,2, \ldots)$ функций вида

$$
\psi_{l}\left(p_{1}, p_{2}\right)=\sum_{k, m=-l}^{l} \psi_{l}^{k, m}\left(\left|p_{1}\right|,\left|p_{2}\right|\right) Y_{k}^{l}\left(\theta_{1}, \varphi_{1}\right) Y_{m}^{l}\left(\theta_{2}, \varphi_{2}\right),
$$

где $\psi_{l}^{k, m}$ - функция из $L_{2}\left(\left(R_{+}\right)^{2}, r^{2} t^{2} d r d t\right) \equiv L_{2}\left(R_{+}, r^{2} d r\right) \otimes L_{2}\left(R_{+}, t^{2} d t\right), Y_{k}^{l}$ и $Y_{m}^{l}$ - сферические функции с угловым моментом $l,\left|p_{j}\right|, \theta_{j}, \varphi_{j}$ - сферические координаты вектора $p_{j}, j=1,2$, и представление группы врашения (22) неприводимо.

Часть оператора $H_{\varepsilon}$, действуюшую в $\aleph_{l}$, обозначим через $H_{\varepsilon}^{(l)}$. Подпространство $\aleph_{0}$ состоит из функций $\psi\left(p_{1}, p_{2}\right)$, не меняющихся при врашениях (22). Часть оператора $H^{*}$, действующую в $\aleph_{l}$, обозначим через $H_{l}^{*}$.

Пусть $W_{l}^{(3)}(l=0,1,2, \ldots)$ - пространство троек функций $\vec{u}(p)=\left\{u_{j}(p)\right\}$, полученное пополнением подпространства $L_{l}$ по скалярному произведению $[\vec{u}, \vec{v}]=(K \vec{u}, \vec{v})$. Часть оператора $A_{\varepsilon}$, действуюшую в $W_{l}^{(3)}$, обозначим через $A_{\varepsilon}(l)$, а часть оператора $K$, действуюшую в $L_{l},-$ через $K_{l}$. Тогда оператор $A_{\varepsilon}(l)$ можно определить следуюшим образом:

$$
A_{\varepsilon}(l)=K_{l}^{-1}\left[T_{l}(-1)+2 \pi^{2} E\right], \quad D\left(A_{\varepsilon}(l)\right)=D\left(T_{l}(-1)\right) \subset W_{l}^{(3)} .
$$

Нетрудно убедиться в том, что область определения $D\left(H_{l}^{*}\right)$ оператора $H_{l}^{*}$ состоит из таких функций $g\left(p_{1}, p_{2}\right)$ вида (5), для которых $f \in D(H) \cap \aleph_{l}, \vec{u}, \vec{v} \in W_{l}^{(3)}$. Аналогично область определения $D\left(H_{\varepsilon}^{(l)}\right)$ оператора $H_{\varepsilon}^{(l)}$ состоит из таких функций $g\left(p_{1}, p_{2}\right)$ вида (5), для которых $f \in D(H) \cap \aleph_{l}, \vec{u}, \vec{v} \in W_{l}^{(3)}$ и $\vec{v}=A_{\varepsilon}(l) \vec{u}$.

ЛЕмма 5. Пусть $A_{\varepsilon}$ - симметрический оператор, его индексы дефекта равньи и совпадают с индексами дефекта оператора $T(-1)$. В частности, $A_{\varepsilon}(0)$ симметрический оператор с индексами дефекта $(1,1)$. 
ДоКАЗАТЕЛЬСТво легко следует из того факта, что квадратичная форма оператора $A_{\varepsilon}$ в $W^{(3)}$ совпадает с квадратичной формой оператора $T(-1)+2 \pi^{2} E$ в $L_{2}^{(3)}$. Действительно,

$$
\left[A_{\varepsilon} \vec{u}, \vec{v}\right]=\left(K K^{-1}\left[T(-1)+2 \pi^{2} E\right] \vec{u}, \vec{v}\right)=\left(\left[T(-1)+2 \pi^{2} E\right] \vec{u}, \vec{v}\right),
$$

где $\vec{u}, \vec{v} \in D(T(-1))$.

Рассмотрим семейство $\left\{A_{\varepsilon \beta}(0)\right\}, \varepsilon \in \mathbb{R}^{3}, \beta \in \mathbb{C},|\beta|=1$, операторов

$$
A_{\varepsilon \beta}(0)=K_{0}^{-1}\left[T_{0 \beta}(-1)+2 \pi^{2} E\right], \quad D\left(A_{\varepsilon \beta}(0)\right)=D\left(T_{0 \beta}(-1)\right),
$$

действуюших в подпространстве $W_{0}^{(3)}$. Поскольку квадратичная форма $\left[A_{\varepsilon \beta}(0) \vec{u}, \vec{v}\right]$ в $W_{0}^{(3)}$ совпадает с квадратичной формой $\left(\left[T_{0 \beta}(-1)+2 \pi^{2} E\right] \vec{u}, \vec{v}\right)$ в $L_{0} \subset L_{2}^{(3)}$, то из самосопряженности оператора $T_{0 \beta}(-1)$ вытекает самосопряженность оператора $A_{\varepsilon \beta}(0)$. Обозначим через $H_{\varepsilon \beta}^{(0)}$ сужение оператора $H_{0}^{*}$ на область $D\left(H_{\varepsilon \beta}^{(0)}\right)$, состоящую из таких функций $g\left(p_{1}, p_{2}\right)$ вида (5), для которых $f \in D(H) \cap \aleph_{0}, \vec{u}, \vec{v} \in W_{0}^{(0)}$ и $\vec{v}=A_{\varepsilon \beta}(0) \vec{u}$. С помошью леммы 3 можно установить, что для любых $\varepsilon \in \mathbb{R}^{3}$ и $\beta \in \mathbb{C},|\beta|=1$, оператор $H_{\varepsilon \beta}^{(0)}$ является самосопряженным.

Лемма 6. Оператор $H_{\varepsilon}^{(0)}$ имеет индексы дефекта $(1,1)$.

ДокАЗАТЕЛЬСтво. Из общей теории самосопряженных расширений симметрических операторов легко следует (см. [2])

ПРЕДЛОЖЕНИЕ. Коразмерность области определения $D(A)$ симметрического оператора А с конечными и равныцми индексами дефекта $(n, n)$ в области $D(\tilde{A})$ любого его самосопряженного расширения равна п. Если индексы дефекта оператора А бесконечны и у него существуют самосопряженные расширения, то найдется такое самосопряженное расширение $\hat{A}$, что $D(A)$ имеет в $D(\hat{A})$ бесконечную коразмерность.

Вернемся к схеме расширения операторов $H_{\varepsilon}$ и $H_{\varepsilon}^{(0)}$. Все самосопряженные расширения оператора $H_{\varepsilon}^{(0)}$ получаются с помошью самосопряженных расширений оператоpa $A_{\varepsilon}(0)$, поэтому они описываются введенным вьшше классом операторов $H_{\varepsilon \beta}^{(0)}$. В силу предложения коразмерность $D\left(A_{\varepsilon}(0)\right)$ в $D\left(A_{\varepsilon \beta}(0)\right)\left(D\left(T_{0}(-1)\right)\right.$ в $\left.D\left(T_{0 \beta}(-1)\right)\right)$ равна единице. Отсюда и из схем расширения оператора $H_{\varepsilon}^{(0)}$ следует, что коразмерность области определения $D\left(H_{\varepsilon}^{(0)}\right)$ оператора $H_{\varepsilon}^{(0)}$ в области определения $D\left(H_{\varepsilon \beta}^{(0)}\right)$ оператора $H_{\varepsilon \beta}^{(0)}$ равна единице. Теперь утверждение леммы следует из предложения. Лемма доказана.

Как и в случае $l=0$, можно показать, что индексы дефекта операторов $H_{\varepsilon}^{(l)}(l \geqslant 1)$ равны и не превышают $(1,1)$. Начиная с некоторого $l_{1}$ (или при $l \geqslant l_{1}$ ) все операторы $H_{\varepsilon}^{(l)}$ самосопряжены.

Таким образом, для любого $\varepsilon \in \mathbb{R}^{3}$ оператор $H_{\varepsilon}$ является симметрическим оператором с ненулевыми, равными и конечными индексами дефекта. 
Из леммы 6 следует, что для любого $z \in \mathbb{C} \backslash \mathbb{R}^{1}$ существует единственное с точностью до умножения на константу решение уравнения

$$
\left(H_{\varepsilon}^{(0)}\right)^{*} g=z g .
$$

Но решение уравнения (24) имеет вид (5) с дополнительными условиями $f \in D(H) \cap \aleph_{0}$, $\vec{u}, \vec{v} \in W_{0}^{(3)}, \vec{v}=A_{\varepsilon}^{*}(0) \vec{u}$. Подставим теперь в уравнение (24) функцию $g$ в форме (5) и воспользуемся видом $\vec{v}=A_{\varepsilon}^{*}(0) \vec{u}$, учитьвая определение $A_{\varepsilon}(0)$. Уравнение $(24)$ с учетом сферической симметричности функций $u_{j}(p)=u_{j}(|p|)=u_{j}(r)(j=1,2,3)$ примет вид

$$
\begin{aligned}
& \pi\left(2 m_{j}\right)^{\frac{3}{2}}\left(\sqrt{\frac{1}{2 m_{k}} r^{2}-z}+\varepsilon_{k}\right) u_{k}(r)=2 \int_{0}^{\infty} \frac{t^{2} u_{j}(t) d t}{\left(2 m_{k}\right)^{-1} r^{2}+\left(2 m_{j}\right)^{-1} t^{2}-z}+ \\
& +m_{j} \int_{0}^{\infty} \frac{t}{r} \ln \left[\frac{\eta^{-1} r^{2}+m_{j}^{-1} r t+\left(2 m_{j}\right)^{-1} t^{2}-z}{\eta^{-1} r^{2}-m_{j}^{-1} r t+\left(2 m_{j}\right)^{-1} t^{2}-z}\right] u_{3}(t) d t, \\
& \quad j, k=1,2, \quad j \neq k, \\
& \pi \eta^{\frac{3}{2}}\left(\sqrt{\left.\frac{r^{2}}{2 M}-z+\varepsilon_{3}\right) u_{3}(r)=}\right. \\
& =\sum_{k \neq j} m_{j} \int_{0}^{\infty} \frac{t}{r} \ln \left[\frac{\left(2 m_{j}\right)^{-1} r^{2}+m_{j}^{-1} r t+\eta^{-1} t^{2}-z}{\left(2 m_{j}\right)^{-1} r^{2}-m_{j}^{-1} r t+\eta^{-1} t^{2}-z}\right] u_{k}(t) d t .
\end{aligned}
$$

Теорема 3. Если $\lambda_{0} \in \mathbb{R} u \lambda_{0}>2 \max _{1 \leqslant j \leqslant 3} \varepsilon_{j}$, mo при $z=-\lambda_{0}^{2}$ система (25) имеет решение.

ДокАЗАТЕЛЬСтво. Выберем $z=-\lambda_{0}^{2}+i \delta, \delta>0, \delta$ мало. При таких $z$ решение системы (25) существует, и из представления

$$
\begin{aligned}
u_{j}(r)= & \pi^{-1}\left(2 m_{k}\right)^{-\frac{3}{2}}\left(\sqrt{\frac{1}{2 m_{j}} r^{2}+\lambda_{0}^{2}-i \delta}+\varepsilon_{j}\right)^{-1} \times \\
\times & \left\{2 \int_{0}^{\infty} \frac{t^{2} u_{2}(t) d t}{\left(2 m_{j}\right)^{-1} r^{2}+\left(2 m_{k}\right)^{-1} t^{2}+\lambda_{0}^{2}-i \delta}+\right. \\
& \left.+m_{k} \int_{0}^{\infty} \frac{t}{r} \ln \left[\frac{\eta^{-1} r^{2}+m_{k}^{-1} r t+\left(2 m_{k}\right)^{-1} t^{2}+\lambda_{0}^{2}-i \delta}{\eta^{-1} r^{2}-m_{k}^{-1} r t+\left(2 m_{k}\right)^{-1} t^{2}+\lambda_{0}^{2}-i \delta}\right] u_{3}(t) d t\right\}, \\
u_{3}(r)= & \pi^{-1} \eta^{-\frac{3}{2}}\left(\sqrt{\frac{1}{2 M} r^{2}+\lambda_{0}^{2}-i \delta}+\varepsilon_{3}\right)^{-1} \times \\
& \times \sum_{j \neq k} m_{k} \int_{0}^{\infty} \frac{t}{r} \ln \left[\frac{\left(2 m_{k}\right)^{-1} r^{2}+m_{k}^{-1} r t+\eta^{-1} t^{2}+\lambda_{0}^{2}-i \delta}{\left(2 m_{k}\right)^{-1} r^{2}-m_{k}^{-1} r t+\eta^{-1} t^{2}+\lambda_{0}^{2}-i \delta}\right] u_{j}(t) d t
\end{aligned}
$$

следует, что его компоненты $u_{j}(r)$ аналитичны в максимальной угловой окрестности положительной вещественной полуоси, ограниченной лучами, проходящими через начало координат и точки $z_{0}^{(j)}$ и $\bar{z}_{0}^{(j)}$, где

$$
z_{0}^{(j)}=2 m_{j}\left(\varepsilon_{j}^{2}-\lambda_{0}^{2}+i \delta\right), \quad j=1,2, \quad z_{0}^{(3)}=2 M\left(\varepsilon_{3}^{2}-\lambda_{0}^{2}+i \delta\right)
$$


(это область, в которой суммы, стоящие в знаменателях в правых частях (26), не обрашаются в нуль).

Сделав в (26) замену $r=\lambda \zeta, t=\lambda \xi, \lambda=\sqrt{-z}$, получим уравнения

$$
\begin{aligned}
u_{j}(\lambda \zeta)= & \pi^{-1}\left(2 m_{k}\right)^{-\frac{3}{2}}\left(\sqrt{\frac{1}{2 m_{j}} \zeta^{2}+1}+\frac{\varepsilon_{j}}{\lambda}\right)^{-1} \times \\
& \times\left\{2 \int_{\gamma_{\xi}} \frac{\xi^{2} u_{k}(\lambda \xi) d \xi}{\left(2 m_{j}\right)^{-1} \zeta^{2}+\left(2 m_{k}\right)^{-1} \xi^{2}+1}+\right. \\
& \left.+m_{k} \int_{\gamma_{\xi}} \frac{\xi}{\zeta} \ln \left[\frac{\eta^{-1} \zeta^{2}+m_{k}^{-1} \zeta \xi+\left(2 m_{k}\right)^{-1} \xi^{2}+1}{\eta^{-1} \zeta^{2}-m_{k}^{-1} \zeta \xi+\left(2 m_{k}\right)^{-1} \xi^{2}+1}\right] u_{3}(\lambda \xi) d \xi\right\}, \\
u_{3}(\lambda \zeta)= & \pi^{-1} \eta^{-\frac{3}{2}}\left(\sqrt{\frac{1}{2 M} \zeta^{2}+1}+\frac{\varepsilon_{3}}{\lambda}\right)^{-1} \times \\
& \times \sum_{j \neq k} m_{k} \int_{\gamma_{\xi}} \frac{\xi}{\zeta} \ln \left[\frac{\left(2 m_{k}\right)^{-1} \zeta^{2}+m_{k}^{-1} \zeta \xi+\eta^{-1} \xi^{2}+1}{\left(2 m_{k}\right)^{-1} \zeta^{2}-m_{k}^{-1} \zeta \xi+\eta^{-1} \xi^{2}+1}\right] u_{j}(\lambda \xi) d \xi
\end{aligned}
$$

(здесь выбрана ветвь $\operatorname{Im} \sqrt{-z}>0$ ). Заметим, что согласно выбору $z$ контур $\gamma_{\xi}$ есть луч, лежаший в области аналитичности функций $u_{j}(\xi)$. Ввиду того что интегралы в правых частях (27) по дуге окружности, соединяюшей контур $\gamma_{\xi}$ и вешественную положительную полуось, стремятся к нулю с ростом радиуса, интегрирование можно проводить по вешественной полуоси. Кроме того, из представления (27) следует сушествование предела $u_{j}(\lambda \zeta)$ при $\zeta \in \mathbb{R}$. Переходя теперь к случаю $\delta=0$ (что возможно в силу аналитичности правых частей (27) по $z$ ), мы приходим к выводу о сушествовании решения системы уравнений

$$
\begin{aligned}
u_{j}\left(\lambda_{0} \zeta\right)= & \pi^{-1}\left(2 m_{k}\right)^{-\frac{3}{2}}\left(\sqrt{\frac{1}{2 m_{j}} \zeta^{2}+1}+\frac{\varepsilon_{j}}{\lambda_{0}}\right)^{-1} \times \\
& \times\left\{2 \int_{0}^{\infty} \frac{\xi^{2} u_{k}\left(\lambda_{0} \xi\right) d \xi}{\left(2 m_{j}\right)^{-1} \zeta^{2}+\left(2 m_{k}\right)^{-1} \xi^{2}+1}+\right. \\
& \left.+m_{k} \int_{0}^{\infty} \frac{\xi}{\zeta} \ln \left[\frac{\eta^{-1} \zeta^{2}+m_{k}^{-1} \zeta \xi+\left(2 m_{k}\right)^{-1} \xi^{2}+1}{\eta^{-1} \zeta^{2}-m_{k}^{-1} \zeta \xi+\left(2 m_{k}\right)^{-1} \xi^{2}+1}\right] u_{3}\left(\lambda_{0} \xi\right) d \xi\right\} \\
& j, k=1,2, j \neq k, \\
u_{3}\left(\lambda_{0} \zeta\right)= & \pi^{-1} \eta^{-\frac{3}{2}}\left(\sqrt{\frac{1}{2 M} \zeta^{2}+1}+\frac{\varepsilon_{3}}{\lambda_{0}}\right)^{-1} \times \\
& \times \sum_{j \neq k} m_{k} \int_{0}^{\infty} \frac{\xi}{\zeta} \ln \left[\frac{\left(2 m_{k}\right)^{-1} \zeta^{2}+m_{k}^{-1} \zeta \xi+\eta^{-1} \xi^{2}+1}{\left(2 m_{k}\right)^{-1} \zeta^{2}-m_{k}^{-1} \zeta \xi+\eta^{-1} \xi^{2}+1}\right] u_{j}\left(\lambda_{0} \xi\right) d \xi
\end{aligned}
$$

Сделав в (28) замену $r=\lambda_{0} \zeta, t=\lambda_{0} \xi$, получим утверждение теоремы. 
СлЕДСтвиЕ. Система (25) при $z=-\lambda_{0}^{2}, \lambda>\lambda_{0}$ имеет решение, которое можно получить из решения системы при $z=-\lambda_{0}^{2}, \varepsilon_{j}^{\prime}=\left(\lambda_{0} / \lambda\right) \varepsilon_{j}$ заменой переменных $r \rightarrow\left(\lambda / \lambda_{0}\right) r, \quad t \rightarrow\left(\lambda / \lambda_{0}\right) t$.

Заметим, что решения системы (25) лежат в области определения $D\left(T_{0}(-1)^{*}\right)$ оператора $T_{0}(-1)^{*}$. Вернувшись к описанию (21) этой области и выполнив обратноепреобразование Меллина, получим, что имеет место представление (с точностью до умножения на константу) решения системы (25) в виде

$$
u_{j}(r)=\frac{e_{j} r^{i s_{0}}}{r^{2}+1}+\frac{c(\varepsilon) \bar{e}_{j} r^{-i s_{0}}}{r^{2}+1}+\widetilde{u}_{j}(r),
$$

где $\vec{e}=\left\{e_{j}\right\}$ - вектор, введенный в доказательстве теоремы 1 (см. (20)), а $\left\{\widetilde{u}_{j}\right\} \in$ $D\left(T_{0}(-1)\right), c(\varepsilon)$ - константа $(|c(\varepsilon)|=1$ в силу вешественности решений $), \varepsilon=\left(\varepsilon_{1}, \varepsilon_{2}, \varepsilon\right) \in$ $\mathbb{R}^{3}$

Лемма 7. Функция с $(\varepsilon)$ непрерывна в нуле по $\varepsilon_{j}, j=1,2,3$.

ДокАЗАТЕЛЬСТво. Из представления (26) следует, что функции $u_{j}(r)$ непрерывны по $\varepsilon_{j}$, следовательно, их преобразования Меллина непрерьвны по $\varepsilon_{j}$. Но они имеют вид (21), и функция $c(\varepsilon)$ есть вычет в точке $s_{0}+i / 2$ фиксированной линейной комбинации преобразований Меллина $g_{j}(s)$ функций $u_{j}(r)$ и, следовательно, непрерывна по $\varepsilon_{j}$. Лемма доказана.

Теорема 4. Самосопряженное расиирение $H_{\varepsilon, \beta}^{(0)}$ оператора $H_{\varepsilon}^{(0)}$ имеет бесконечный набор отрицательных собственных значений, уходящих на $-\infty$.

ДокАЗАТЕЛЬСтво. В силу теоремы 2 область определения $D\left(T_{0, \beta}(-1)\right)$ оператора $T_{0, \beta}(-1)$ состоит из троек вида $\vec{u}(r)=\left\{u_{j}(r)\right\}_{1 \leqslant j \leqslant 3}$,

$$
u_{j}(r)=\frac{e_{j} r^{i s_{0}}+\beta \bar{e}_{j} r^{-i s_{0}}}{r^{2}+1}+\widetilde{u}_{j}(r), \quad\left\{\widetilde{u}_{j}(r)\right\} \in D\left(T_{0}(-1)\right) .
$$

Найдем собственную функцию оператора $H_{\varepsilon \beta}^{(0)}$, отвечаюшую собственному значению $-\lambda^{2}$. Нетрудно видеть, что ее можно получить в виде (5) с учетом условия $\vec{v}=A_{\varepsilon, \beta}(0) \vec{u}$ из решения $\left\{u_{j}(r)\right\}$ системы (25) при $z=-\lambda^{2}$ с дополнительным условием $\left\{u_{j}(r)\right\} \in$ $D\left(T_{0, \beta}(-1)\right)$. Это значит, что функции $u_{j}(r)$ должны удовлетворять представлению $(30)$

В силу следствия теоремы 3 и леммы 6 при достаточно больших $\lambda$ условие (30) равносильно условию

$$
c(0)\left(\frac{\lambda}{\lambda_{0}}\right)^{2 i s_{0}}=\beta,
$$

где $c(0)=\lim _{\varepsilon \rightarrow 0} c(\varepsilon), \lambda_{0}$ - некоторое фиксированное число, при котором система (25) при $z=-\lambda_{0}^{2}$ имеет решение. Но условию (31) удовлетворяет бесконечный набор значений

$$
\lambda_{n}=\lambda_{0} \exp \left(\frac{\arg (\beta / c(0))}{2 s_{0}}+\frac{\pi n}{s_{0}}\right), \quad n=0,1,2, \ldots
$$


Таким образом, мы получили бесконечный набор собственных значений $z_{0}=-\lambda_{n}^{2}$, уходящих на $-\infty$. Теорема доказана.

Поскольку для любого $\varepsilon \in \mathbb{R}^{3}$ симметрический оператор $H_{\varepsilon}$ имеет ненулевые, равные и конечные индексы дефекта, он обладает самосопряженными расширениями. Предположим, что $\widehat{H}_{\varepsilon}$ - некоторое самосопряженное расширение оператора $H_{\varepsilon}$. Тогда $\widehat{H}_{\varepsilon}^{(0)}$ - часть оператора $\widehat{H}_{\varepsilon}$, действуюшая в $\aleph_{0}$, - является самосопряженным расширением оператора $H_{\varepsilon}^{(0)}$. Так как самосопряженные расширения оператора $H_{\varepsilon}^{(0)}$ исчерпьваются семейством операторов $\left\{H_{\varepsilon, \beta}^{(0)}\right\}$, то найдется некоторое значение $\beta \in \mathbb{C}(|\beta|=1)$ такое, что $\widehat{H}_{\varepsilon}^{(0)} \equiv H_{\varepsilon, \beta}^{(0)}$. Следовательно, самосопряженный оператор $\widehat{H}_{\varepsilon}$ имеет бесконечный набор отрицательных собственных значений вида (32), уходящих на $-\infty$.

ТЕОРема 5. Любое самосопряэсенное расиирение $\widehat{H}_{\varepsilon}$ оператора $H_{\varepsilon}$ имеет бесконечный набор отрицательных собственных значений, уходящих на $-\infty$.

Благодарности. Автор выражает особую признательность Р. А. Минлосу за постоянное внимание и ценные советы. Работа поддержана Фондом фундаментальных исследований АН РУз.

\section{Список литературы}

[1] Ф. А. Березин, Л. Д. Фаддеев. ДАН СССР. 1961. Т. 137. № 5. С. 1011-1014.

[2] Н. И. Ахиезер, И. М. Глазман. Теория линейных операторов в гильбертовом пространстве. М.: Наука, 1966.

[3] Р. А. Минлос, Л. Д. Фаддеев. ДАН СССР. 1961. Т. 141. № 6. С. 1335-1338.

[4] Р. А. Минлос, Л. Д. Фаддеев. ЖЭТФ. 1961. Т. 41. № 12. С. 1850-1851.

[5] Г. В. Скорняков, К. А. Тер-Мартиросян. ЖЭТФ. 1956. Т. 31. № 5. С. 775-790.

[6] Г. С. Данилов. ЖЭТФ. 1961. Т. 40. № 2. С. 498-507.

[7] Р. А. Минлос, М. Х. Шерматов. Вестн. МГУ. Сер. 1. 1989. № 6. С. 7-14.

[8] A. M. Mel'nikov, R. A. Minlos. Adv. Sov. Math. 1991. V. 5. P. 99-112.

[9] М. ШІ. Бирман. Матем. сб. 1956. Т. 38. № 4. С. 431-450.

[10] И. М. Гельфанд, Р.А. Минлос, З. Я. Шапиро. Представление группы вращений и группы Лоренца, их применения. М.: Физматгиз, 1958.

[11] К. Гофман. Банаховы пространства аналитических функций. М.-Л.: ИЛ, 1963.

Поступила в редакцию 20.III.2000 г. 